\title{
Una mirada en blanco y negro: resignificación de la fotografía*
}

\section{Resumen}

En este artículo se reflexiona alrededor de las prácticas pedagógicas a partir de un análisis de experiencia de aula, asociado a la enseñanza de la fotografía del programa de Diseño Gráfico de la Universidad de Boyacá. Por ello, el aula como sitio dentro del cual se ha venido enseñando sobre la importancia del rescate de una técnica artesanal, como lo es la "goma bicromatada", en la clase de fotografía digital y análoga con los estudiantes de quinto semestre.

Se aborda la investigación cualitativa, utilizando el estudio de casos con un grupo focal de 15 estudiantes universitarios. Se buscó evidenciar el uso de técnicas tradicionales de la fotografía como registró tanto artístico, como visual; en el estudio de la imagen así como los alcances estéticos y gráficos.

Se evidenció en el análisis realizado la importancia de las dinámicas asociadas a la apropiación de técnicas tradicionales y la resignificación de la fotografía de mediados del siglo XIX, en la construcción de conocimiento y del lenguaje plástico y visual para el profesional en diseño.

\author{
Sandra Yaneth Chaparro \\ Cardozo. \\ Magíster en Educación. Grupo \\ de investigación LERD, docente \\ tiempo completo, Fundación \\ Universitaria Juan de Castella- \\ nos. Tunja, Colombia. \\ Correo electrónico: sandraya- \\ nethchaparro@gmail.com
}

Recibido: Agosto 2014 Aprobado: Marzo 2015

Palabras clave:

Aprendizaje, enseñanza, fotografía, pedagogía, práctica.

* Artículo de reflexión derivado de investigación. 


\section{A look in black and white: redefinition of photography}

\begin{abstract}
This article presents a reflection about teaching practices from an analysis of classroom experience associated with the teaching of photography in the Graphic Design program at Universidad de Boyacá. This is the reason of the classroom as the place where classes about the importance of rescuing a craft technique such as the "gum bichromate" in the digital and analog photography class with fifth semester students has been taking place.
\end{abstract}

Qualitative research is addressed using case studies with a 15 university students focus group. The purpose was to demonstrate the use of traditional techniques of photography as both artistic and visual records, in the study of image as well as the aesthetic and graphic scope.

In the analysis carried out, the importance of dynamics associated to traditional techniques of appropriation and the redefinition of the mid-ninetienth century photography in building knowledge and the plastic and visual language for professional design were evident.
Key words:

Practice, pedagogy, learning, teaching, photography. 


\section{Introducción}

En el proceso de indagación se abordan las prácticas pedagógicas a partir de una experiencia de aula respondiendo a: ¿Cómo entender los estilos de práctica? ¿Qué se busca con la imagen? Y ¿Cómo el estudiante reinterpreta el mundo mediante la fotografía? De esta forma, la enseñanza de la fotografía análoga dentro del currículo de Diseño Gráfico de la Universidad de Boyacá en la formación de profesionales en el campo del lenguaje visual en el ámbito del diseño.

El lenguaje visual como parte de la comunicación visual a través de la fotografía a modo de instrumento y herramienta de enseñanza en el aula; permitiéndole al estudiante desarrollar un lenguaje y pensamiento en relación a propuestas gráficas y estéticas. La composición de la imagen permitió generar en el estudiante la sensibilidad de la plástica y lo artístico, además de la relación con la exploración de técnicas tradicionales de la fotografía en la producción y el uso de la imagen publicitaria, producto, artística, de moda y editorial, entre otros.

En tanto, la captura de la imagen no solo como un saber que se halla en lo tradicional y que es parte de la historia; la imagen fotográfica es visualizada como pieza fundamental de la academia, en este caso en particular sobre la enseñanza desde el lenguaje visual. En la formación de profesionales en el campo del diseño y la comunicación visual, se permite establecer de qué manera se sitúa la práctica pedagógica y los aprendizajes de los estudiantes de Diseño Gráfico con relación a la fotografía. La experiencia de aula como dispositivo de indagación permite potenciar en el estudiante elementos no solo históricos y técnicos. Se abordó el lenguaje visual mediante la técnica de la fotografía tradicional "goma bicromatada" dentro de un proceso histórico, técnico y conceptual de la fotografía desde su invención hasta nuestros días entendiendo la imagen como parte de una representación visual y el desarrollo estético. 


\section{Metodología}

Este estudio nace de la resignificación de técnicas tradicionales de la fotografía, como la "goma bicromatada", en una experiencia de aula. Se buscó evidenciar el uso de técnicas tradicionales de la fotografía como registró tanto artístico, como visual; mediante el análisis de los procesos en la apropiación de la imagen, así como los alcances estéticos y gráficos que le aportan a la academia.

Mediante un enfoque cualitativo y un tipo de estudio de casos, se usaron técnicas como la observación participante y el diario de campo como instrumento de análisis y recolección de información de una población de muestra en un grupo focal de 15 estudiantes universitarios. Se utilizó la metodología pedagógica lo experiencial y se abordó análisis de la práctica pedagógica y el impacto en relación de las técnicas y saberes frente la imagen fotográfica en un proceso de enseñanza y aprendizaje.

En un ejercicio de experiencia de aula en la praxis o práctica en la academia, a partir de la práctica docente, esta investigación surge de una necesidad de rescatar la fotografía tradicional como dispositivo de aprendizaje en el lenguaje visual; producto de la experiencia de la práctica pedagógica se usó el diario de campo, se recopilaron experiencias y opiniones de los estudiantes frente a la fotografía como dispositivo de aprendizaje mediante el análisis y la observación participante, se analizó la importancia de las prácticas pedagógicas y la necesidad de sistematízalas.

Con el análisis sistemático, se encontraron aspectos en los que se hace necesaria la enseñanza de la fotografía tradicional en el currículo de Diseño Gráfico, dado que permite potenciar elementos de la fotografía digital. Con ello, potenciar las técnicas tradicionales como parte del desarrollo y el intercambio de saberes con un grupo de estudiantes; allí se analizó la importancia de las prácticas pedagógicas y la importancia del rescate de técnicas ya olvidadas en la academia. 


\section{Discusión}

\section{Pensar en blanco y negro: de la práctica a la experiencia con la fotografía}

La fotografía como instrumento en la reproducción de realidades congeladas en el tiempo, como resultado una imagen que cuenta historias familiares, acontecimientos políticos y violentos. En el estudio se describe la práctica pedagógica a través de una experiencia de aula producto del análisis de la asignatura de fotografía. En el estudio se interpreta la imagen como dispositivo de producción gráfica y visual; por tanto, el aula como escenario y como sitio, en el que se han adelantado procesos pedagógicos alrededor de habilidades y métodos en los que se identificó la importancia de la imagen publicitaria y editorial, entre otros.

Describir la práctica pedagógica a partir de la fotografía permite interpretar la experiencia de aula tanto en la fotografía como parte de los contenidos de Diseño Gráfico, primando la capacidad para reconocer las herramientas, técnicas y conceptos del lenguaje fotográfico y publicitario, por ello es fundamental en la formación de profesionales de esta disciplina; entre tanto, el currículo está permeado por las prácticas pedagógicas, la enseñanza, aprendizaje y en general por todo aquello que suceda en el aula. En el desarrollo de una clase son parte fundamental los espacios, tiempos, objetos, situaciones, que aunque sean irrisorios cobran sentido; como lo indica Jackson (1992): "Para apreciar el significado de los hechos triviales del aula, es necesario considerar la frecuencia de su apropiación, la uniformidad del entorno escolar y la obligatoriedad de la asistencia diaria" (p. 4).

Parte de los aprendizajes es no solo reconocer los conceptos de la fotografía y las técnicas que obedecen a la captura de un instante de realidad. Entre tanto, la imagen fotográfica es pintar con la luz y proviene del griego phos (luz) y grafis (diseñar, escribir y pintar).

'La práctica proviene de 'praxis' que significa el hacer a través de una serie de habilidades cuyo fin es la innovación o transformación de una comunidad. 
Si la luz constituye el material básico del fotógrafo, las sustancias sensibles a la luz van a proporcionarle su soporte primario. El soporte fotosensible da a la fotografía su carta de naturaleza; es decir, constituye la sola condición sine qua non para que exista fotografía. En la mayoría de los manuales, la fotografía se describe como una interacción entre un dispositivo óptico (la cámara) y una superficie sensible; aquí en cambio se defiende la hegemonía de este segundo competente, ya que la cámara no es más que un accidente histórico en el proceso fotográfico. (Fontcuberta, 1994, p 41.)

La imagen se fija como una proyección de la luz utilizando unas técnicas que por años se han venido modificando. Trayendo a este estudio uno de los procedimientos elaborados por Niépce como lo es la heliografía como parte de la representación gráfica; por otro lado Daguerre, uno de los fotógrafos más conocidos dentro de la historia, junto con un proceso de captura y copia de la luz, el Daguerrotipo; como parte de las praxis de aquel momento en relación a la fotografía surge Fox Talbot, generando ciertos estudios en relación al problema de positivo y negativo, popularmente conocido como la calotipia, que parte de lo bello y su forma.

Atendiendo a la importancia y la pertinencia en la resignificación de las diferentes tendencias de la fotografía tradicional, así como a la importancia de la praxis dentro de la academia, -entre tanto la técnica de la "goma bicromatada" - rescatada por Joan Fontcuberta. Este tipo de fotografía tradicional se desarrolló como técnica de impresión fotográfica, en un proceso en que se hace necesario el uso de goma arábiga y bicromato de potasio, que se utilizó a finales de 1800. Esta técnica fotográfica tradicional se desarrolló con John Pouncy en 1858.

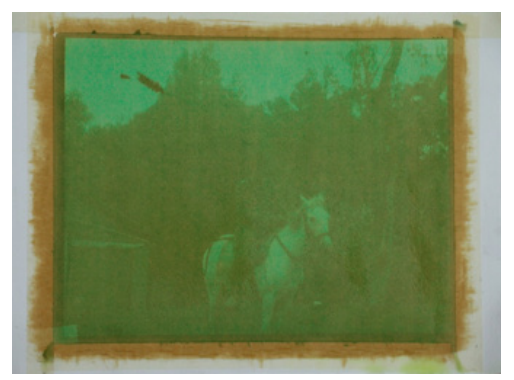

Figura 1. Técnica de fotografía artesanal "goma bicromatada". Imagen de Sebastián Arguello. ${ }^{2}$ Fuente propia: Sandra Yaneth Chaparro C. 
En la Figura 1 se desarrolla la técnica de la "goma bicromatada", usando pigmentos solubles mezclados con goma arábiga y bicromato; en el desarrollo de esta técnica se habla de Robert Demachy en 1859. La fotografía, durante diferentes momentos históricos, es la captura de una realidad en una imagen como producto del empirismo usando la técnica de la goma arábiga y el bicromato de potasio. El uso de las técnicas tradicionales en la academia permite visualizar la fotografía como un elemento creativo dentro de las prácticas pedagógicas y el desarrollo de los estilos de aprendizaje.

Dentro de la práctica pedagógica el eje es la fotografía y el sitio es el aula, donde se desarrollaron procesos histórico-creativos de la imagen, visualizando la tecnología a partir de lo tradicional. La técnica de nobles de Pouncy dentro del currículo de Diseño Gráfico, le otorga a la práctica pedagógica un afianzamiento en las aptitudes para el desempeño del lenguaje y la comunicación visual. Para ello, Berger (1989) anota en relación a la imagen y la publicidad que "[...] toda imagen publicitaria confirma y apoya a las demás. La publicidad no es simplemente un conjunto de mensajes en competencia; es un lenguaje en sí misma, que se utiliza siempre para alcanzar el mismo objetivo general" (p. 145). La imagen publicitaria que parte, del hacer de la fotografía, y que representa el lenguaje visual dentro la asignatura de fotografía digital y análoga; entre tanto, la fotografía parte de la apropiación y adquisición de experiencias dentro de la práctica pedagógica y la exploración de los diferentes estilos de aprendizajes.

La práctica hace parte de la construcción de los estilos de aprendizaje del estudiante, en el ejercicio de la praxis la producción de imagen publicitaria como eje fundamental en la formación de profesionales en el uso de herramientas tradicionales, tecnológicas y visuales. "Por tanto, el conocimiento de los procesos de asimilación (estilos de aprendizaje), así como los métodos o formas de experimentar esa información (didáctica), implican un desarrollo intelectual y de aprendizaje que podría orientarse hacia la capacitación creativa (creatividad)" (Suárez, 2012, p. 4). 
La didáctica como instrumento mediante el cual se fortalece el estilo de aprendizaje de los estudiantes, así como la apropiación de la imagen publicitaria en el entorno profesional. El docente es quien construye a través de la experiencia de aula, estilos de prácticas en el aprendizaje de la imagen fotográfica en el sitio profesional.

La tendencia al desarrollo de la [...] capacidad creativa en el contexto universitario está tomando una gran importancia, siendo una de las competencias a desarrollar en los estudiantes. El conocimiento de formas o modos para potenciarla mediante el aprendizaje es la línea de investigación que desde este trabajo se expone, haciendo hincapié en la importancia de conocer los estilos de aprendizaje de nuestros estudiantes universitarios para desarrollar didácticamente su capacidad creativa. (Suárez, 2012, p. 3)

La experiencia de aula, es una constante en la calidad de los aprendizajes del estudiante, por tanto la fotografía como puente en la innovación y creación. Los estilos de la práctica pedagógica se reproducen en el aprendizaje del estudiante; para adquirir un estilo de aprendizaje autónomo a través de lo digital y el uso de diferentes técnicas de la fotografía. "El estilo de pensamiento o estilo intelectual es, pues, como una especie de autogobierno mental, centrado más en los usos que en los niveles de inteligencia; lo que se valora no es cuánta inteligencia tiene una persona, sino cómo la emplea" (Rojas, Salas y Jiménez, 2006, p. 8).

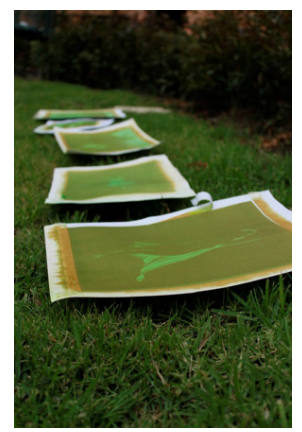

Figura 2. Técnica de fotografía artesanal "goma bicromatada". Imagen de Sandra Yaneth Chaparro. ${ }^{3}$ Fuente propia: Sandra Yaneth Chaparro C. 
El estudiante aprende de forma guiada y autónoma en la construcción de un estilo de aprendizaje a través del estilo de práctica docente; se indaga al estudiante desde interrogantes del saber aprender: ¿Cómo se aprende? ¿Por qué es importante? Y finalmente: ¿Para qué sirve al profesional del diseño?

Estilo de aprendizaje es un término genérico, un concepto paraguas, y un nombre para reconocer las diferencias individuales de aprendizaje. Estilo de aprendizaje ha llegado a ser también un complejo campo de estudio. Así como podemos identificar escuelas de sicología examinando sus opiniones filosóficas y subsecuentes estrategias de operación e implementación, así también podemos identificar diversas aproximaciones al estilo de aprendizaje... Nadie puede reclamar que representa el estilo de aprendizaje en su totalidad. (Butler 1987: 9). (Rojas et al., 2006, p. 6)

Finalmente, las prácticas como experiencia de aula permiten que el estudiante desarrolle habilidades y aprendizajes de forma autónoma. La cátedra de fotografía está orientada a partir de tendencias en la construcción de la práctica y la didáctica pensada para el estudiante; identificado desde la praxis los estilos de aprendizaje en el proceso de la enseñanza en la práctica pedagógica:

[...] la fotografía, como elemento transmisor de información (visual), sería fuente histórica, al ser el documento fotográfico un fragmento (congelado) de la Historia, y lo que es más importante, su valor fontal es intrínseco al ser coetánea del hecho del cual da testimonio. (Lara, 2005, p. 9).

Entre tanto, el aprendizaje es propiciado por el estudiante quien genera propuestas de fotografía en el campo de la publicidad, promoviendo experiencias significativas con el saber hacer y saber crear. Por otra parte, la experiencia de aula en la educación superior como elemento de investigación se transmite una fuente de información visual en la experiencia con el uso de materiales fotosensibles a través de la exploración en el manejo de luz, la composición y los planos. 


\section{Conclusiones}

Este escrito es producto de la experiencia de aula y la reflexión de práctica pensando en blanco y negro mediante estrategias, didácticas, técnicas digitales y tradicionales el desarrollo de la técnica y la exploración con la imagen fotografía. Esta experiencia de aula nace desde la práctica personal en el estudio de técnicas, recursos y la exploración de la técnica de la fotografía "goma bicromatada" en los laboratorios de la clase de fotografía digital y análoga del programa de Diseño Gráfico; permitió generar futuras propuestas digitales a blanco y negro en respuesta al lenguaje visual.

Para este estudio, en particular, la goma como medio cuasi digital dentro de otra época es rescatada como técnica tradicional dándole un valor estético y plástico para el diseño, teniendo en cuenta que para los estudiantes es novedosa y les genera curiosidad tanto en los materiales como en sus resultados finales. Dado que esta técnica requiere de laboratorio de tiempo en el sensibilizado del papel, el revelado y el copiado, esta experiencia permite rescatar el valor de la fotografía como hacer, como vivencia y como experiencia.

La experiencia y la apropiación del conocimiento permite crear un nuevo valor agregado de la fotografía tradicional, cuando se genera toda una construcción de autoaprendizaje con el conocimiento en ese ensayo y error en la experiencia con los materiales y los diversos resultados; la historicidad permite crear todo un lenguaje plástico y visual, mediante el cual se espera que el estudiante en la exploración identifique técnicas tradicionales. En el estudio se identificó que los estudiantes han venido manifestando su interés por estas técnicas tradicionales y un tanto artesanales en el hacer, dada esta condición, para ellos es novedoso ver una fotografía de características particulares. 
La cátedra no solo se trata de una clase magistral o de actividades sometidas a un procedimiento específico, un primer inicio es el aprendizaje de las técnicas tradicionales, en las cuales el estudiante se enfrenta a los emulsionantes fotosensibles, a estar en un laboratorio de mediados de siglo. El estudiante comprende elementos de la fotografía tradicional teniendo en cuenta las transformaciones y tendencias de la imagen publicitaria.

En este estudio se buscó evidenciar el uso de técnicas tradicionales de la fotografía como registro artístico y visual, además de indagar en los estudiantes y en el interior de la práctica docente la posibilidad estética de un lenguaje visual. Mediante la apropiación del conocimiento fotográfico el estudiante crea un valor emocional y creativo sobre la imagen generada, así como la posibilidad estética, técnica y artística que dan pie a la forma, al color y al concepto de blanco y negro en el interior de la academia universitaria mediado en la experiencia.

Es importante que el estudiante descubra la necesidad por el rescate de técnicas como la "goma bicromatada", en el registro de imágenes; teniendo en cuenta la apropiación de la fotografía en el aprendizaje y en la formación profesional. El registro del diario de campo, permitió un acercamiento a la apropiación de la fotografía del lenguaje visual en el diseño gráfico, así como la visión individual de cada estudiante frente a la imagen latente; por otra parte, la calidad de la experiencia en el registro fotográfico. Mediante la clase de fotografía se buscó generar un interés por el desarrollo de competencias en el saber hacer y saber saber en la resignificación de la fotografía tradicional en la implementación de estrategias, recursos, técnicas y estudio documental.

En el trabajo que se ha venido adelantado dentro las clases de fotografía análoga y digital, analizaron experiencias de aula en las que se aborda la práctica docente mediada por enseñanza de la imagen fija en el proceso de la fotografía tradicional, contribuyendo a que el futuro diseñador desarrolle un aprendizaje sobre la luz y 
sus propiedades. La experiencia de aula, evidenció la necesidad de buscar espacios en los cuales se propicie la enseñanza de estas técnicas, favoreciendo actividades didácticas, que permitan generar espacios de aprendizaje dinámicos en relación a la fotografía, en el uso de emulsionantes fotosensibles antiguos y modernos.

De esta forma, se sistematiza la práctica pedagógica, dado que en la educación actual se busca rescatar estas experiencias mediante el desarrollo de estrategias didácticas, en las cuales el estudiante construye un aprendizaje de manera autónoma en la experiencia con el conocimiento de la fotografía tradicional. En el análisis, se encontró que la fotografía tradicional en el currículo de Diseño Gráfico es vital, dado que permite potenciar elementos de la fotografía digital y con ello potenciar las técnicas ya olvidadas y potenciarlas en la academia.

\section{Referencias}

Berger, J. (1989). Modos de ver. Barcelona: Gustavo Gili, S.A.

Fontcuberta, J. (1994). Fotografía: conceptos y procedimientos. Una propuesta fotográfica. México: Editorial Gustavo Gili, S.A.

Jackson, P. W. (1992). La vida en las aulas. Madrid: Ediciones Morata, S.A.

Lara, E. L. (2005). La fotografía como documento histórico-artístico y etnográfico: una epistemología. Revista de Antropología Experimental, 5, 1-28. Recuperado de http://www.bio-design.com.ar/2-UNLa/historia2/libros/fotog\%20documento.pdf

Rojas, G., Salas, R., \& Jiménez, C. (2006). Estilos de aprendizaje y estilos de pensamiento entre los estudiantes universitarios. Estudios Pedagógicos, 32(1), 49-75. 
Chaparro / Una mirada en blanco y negro: resignificación de la fotografía

Suárez, E. M. (2012). Interacción de la creatividad con los estilos de aprendizaje en los estudiantes universitarios de pedagigia de Galicia. REICE, 10(2), 180-200.

Cómo citar este artículo:

Chaparro, S. Y. (2015). Una mirada en blanco y negro: resignificación de la fotografía. Revista Kepes, $11,163-175$. 